\title{
Observation of diffusion and drift of the negative trions in monolayer $\mathrm{WS}_{2}$
}

\author{
Guanghui Cheng, ${ }^{\dagger, \uparrow, \perp}$ Baikui Li, ${ }^{\ddagger}, *$ Zijing Jin, ${ }^{\dagger}$ Meng Zhang, ${ }^{\ddagger}$ Jiannong Wang, ${ }^{\dagger}, *$
}

Department of Physics, Hong Kong University of Science and Technology, Clear Water Bay, Kowloon, Hong Kong

\#ey Laboratory of Optoelectronic Devices and Systems of Ministry of Education and Guangdong Province, College of Physics and Optoelectronic Engineering, Shenzhen University, Shenzhen 518060, Guangdong, China

\Department of Physics and Astronomy, Purdue Quantum Science and Engineering Institute, Purdue University, West Lafayette, Indiana 47907, USA

${ }^{\perp}$ WPI Advanced Institute for Materials Research (AIMR), Tohoku University, Sendai 980-8577, Japan

*To whom correspondence should be addressed:

Baikui Li, Jiannong Wang

E-mails: libk@szu.edu.cn,phjwang@ust.hk 
Note 1. Device preparation and PL spectroscopy

Device preparation. The monolayer $\mathrm{WS}_{2}$ flake was mechanically exfoliated onto $\mathrm{SiO}_{2} / \mathrm{Si}$ wafer from the bulk crystal, confirmed by optical contrast and atomic force microscope. Then it was covered by a thin hexagonal boron nitride (h-BN) flake using the dry transfer technique described in our previous work ${ }^{1}$. The $\mathrm{Au} / \mathrm{Cr}(50 \mathrm{~nm} / 5 \mathrm{~nm})$ electrodes were fabricated using a standard e-beam lithography.

PL spectroscopy. The h-BN/ $\mathrm{WS}_{2} / \mathrm{SiO}_{2}$ device was placed in a helium cryostat which was mounted under an Olympus microscope with a 100× long-working-distance objective. A frequency-doubled Ti: sapphire laser with 200 -fs pulses, $76-\mathrm{MHz}$ repetition rate, $50-\mu \mathrm{W}$ average power and 3.1-eV photon energy was focused to excite excitonic states in the monolayer $\mathrm{WS}_{2}$. The spatially- and time-resolved PL images and spectra were recorded using a Hamamatsu streak camera system. For measuring the spatially-resolved PL, the grating of the spectrometer in front of the streak camera was tuned to $0^{\text {th }}$ order and served as a reflecting mirror $^{2}$. The calibration of pixel/space scaling was performed according to the full width at half maximum (FWHM) of the laser spot $(0.88 \mu \mathrm{m})$. A Keithley $2636 \mathrm{~B}$ was employed to apply bias voltages.

Note 2. Estimation of the quantum degeneracy temperature of trions

The classical Einstein relation may not be applicable here because it requires that the exciton temperature should be larger than the quantum degeneracy temperature $T \gg T_{0} . T_{0}$ is defined as $T_{0}=\left(\pi \hbar^{2} n_{x}\right) / 2 k_{B} M_{x}{ }^{3}$, where $n_{x}$ is the exciton density and $M_{x}$ is the exciton effective mass. For our trion system, we use $M_{x}=$ $0.67 m_{0}{ }^{2}$ and $n_{x}=2 \times 10^{12} \mathrm{~cm}^{-2}$ assuming that $\sim 10 \%$ absorption of incident photons and about $1 / 10$ trions left after rapid recombination in the first 100 ps. $T_{0}$ is then estimated to be $\sim 40 \mathrm{~K}$. Our experimental temperature $T \sim 6 \mathrm{~K}<<T_{0}$.

Moreover, the bias- or field-dependent drift velocity cannot be extracted reliably due to the large error in the linear region with different bias voltages (Figure 3e). Therefore, the mobility, which is the slope of 
the drift velocity as a function of the electric field ${ }^{4}$, cannot be obtained from the bias-dependent drift measurements.
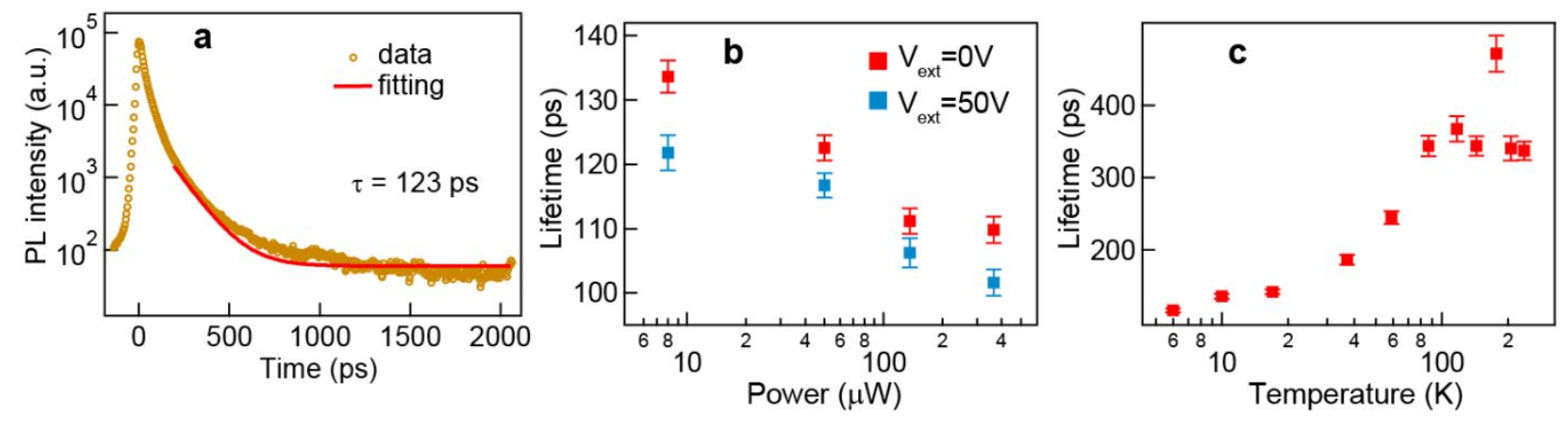

Figure S1. Power and temperature dependence of the lifetimes of long-lived trions. (a) Typical decay curve of the PL intensity of the trion emission and the fitting curve extracted in the time range from 200 ps to 2000 ps using a single exponential function. (b) The lifetime of the long-lived trions as a function of excitation powers under applied bias voltages of $0 \mathrm{~V}$ and $50 \mathrm{~V}$, respectively. The decrease of lifetime at higher power is due to the higher recombination rate of trions when increasing the trion population at higher laser power. The shortened lifetime after bias applied indicates the field-enhanced dissociation of trions. (c) The lifetime of the long-lived trions as a function of temperature.

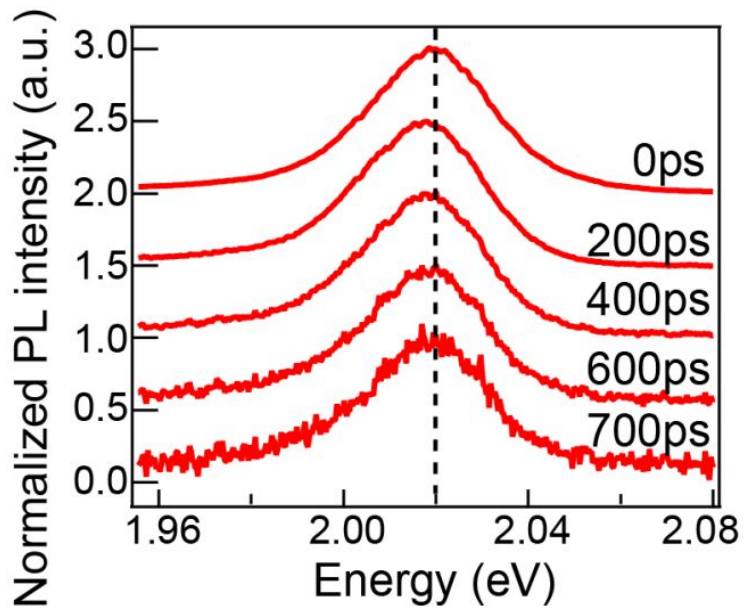


Figure S2. The time-resolved PL spectra. Unchanged peak shape indicates the pure trions emission during nanoseconds time scale. The dashed line is a guide to the eyes. A tiny redshift of emission energy is observed, in consistence with the weakening repulsive dipole interaction as decreasing of the trion density.

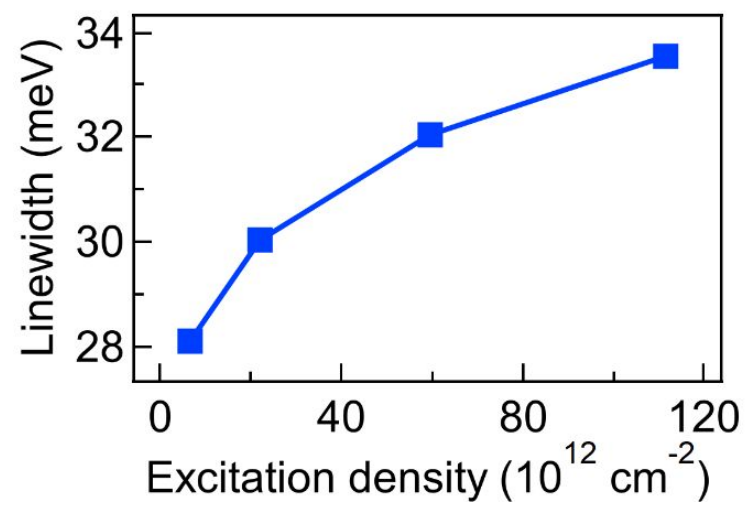

Figure S3. Excitation density dependence of trion emission linewidth. The linewidth follows sublinear dependence, suggesting the trion density is at a high level ${ }^{5-7}$. Such collision-induced spectral broadening indicates a scenario with strong trion-trion and trion-electron scatterings in our device. The excitation density is converted from laser power assuming $10 \%$ absorption of the monolayer $\mathrm{WS}_{2}$ at the laser energy.

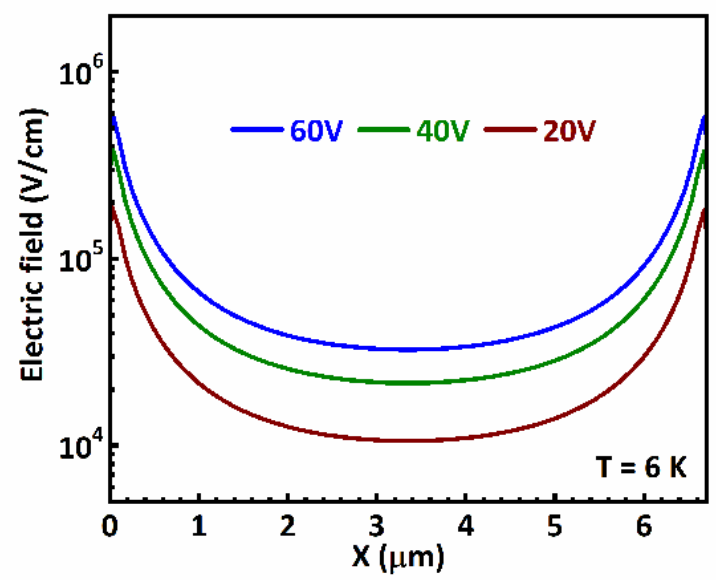

Figure S4. Electric field distribution in the monolayer $\mathrm{WS}_{2}$. Simulation is based on Sentaurus technology computer-aided design (TCAD) and the device configuration in the main text. $\mathrm{X}=0 \mu \mathrm{m}$ is set at the right side of the left electrode. 


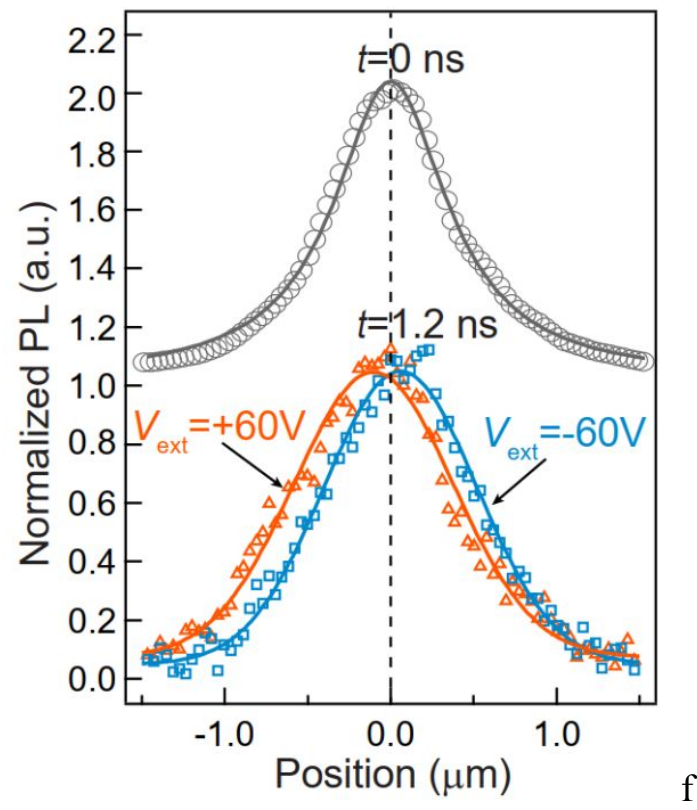

Figure S5. Spatial profiles at $t=0 \mathrm{~ns}$ and $1.2 \mathrm{~ns}$ under voltage $V_{\mathrm{ext}}=60 \mathrm{~V}$ and $-60 \mathrm{~V}$, respectively. Symbols are acquired data. Solid curves are the fittings using the Gaussian function.

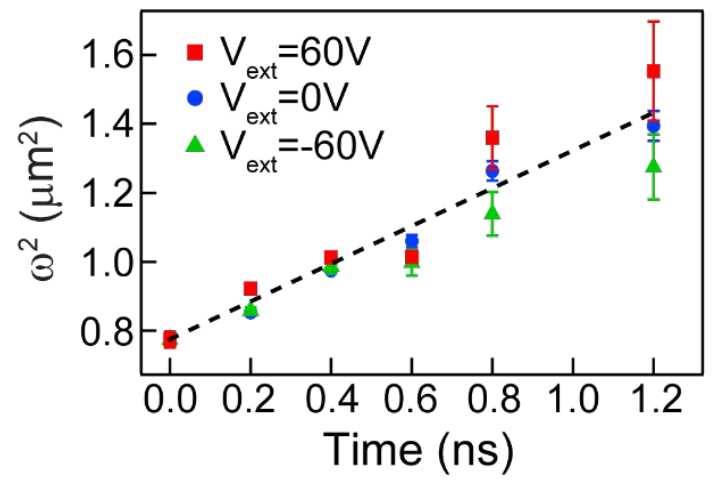

Figure S6. Independence of diffusion on the applied bias voltages. Squared FWHM as a function of decay time under different bias voltages of $0 \mathrm{~V}, 60 \mathrm{~V}$ and $-60 \mathrm{~V}$, respectively. The dashed line is a guide to the eyes. No distinguishable change of the slope indicates the profile broadening and the corresponding diffusion coefficient do not change with different applied voltages. 


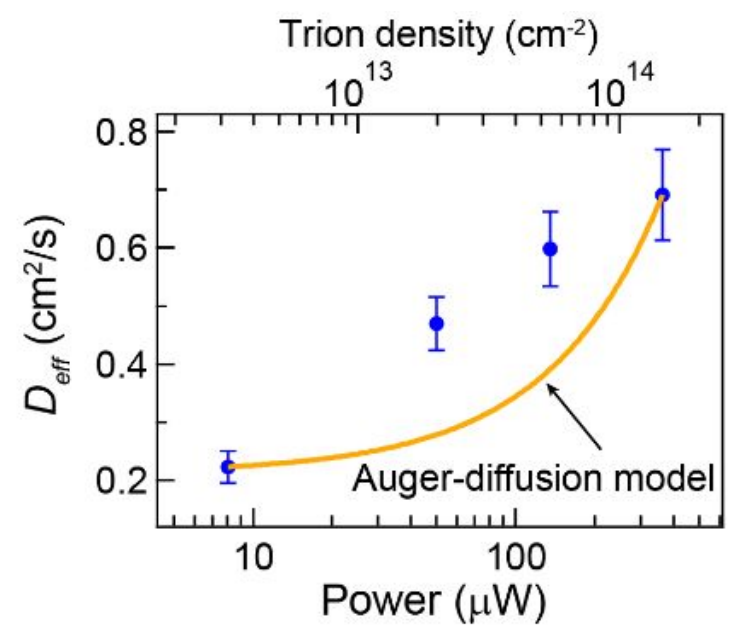

Figure S7. Excitation power dependence of the effective diffusion coefficient $\left(D_{\text {eff }}\right)$ extracted from the broadening of the spatial profiles. The trion densities are extracted assuming $10 \%$ photons converted to trions. The orange line is the fitting by the Auger-diffusion model.

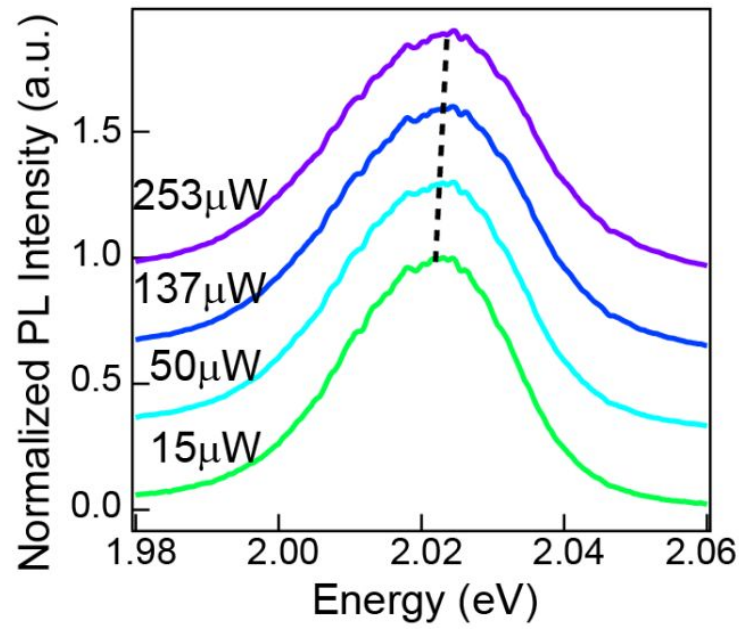

Figure S8. Steady-state PL spectra at different excitation powers. A blueshift is observed with increasing excitation power due to the dipole-dipole interaction of trions in monolayer $\mathrm{WS}_{2}$. The dashed line is a guide to the eyes. 


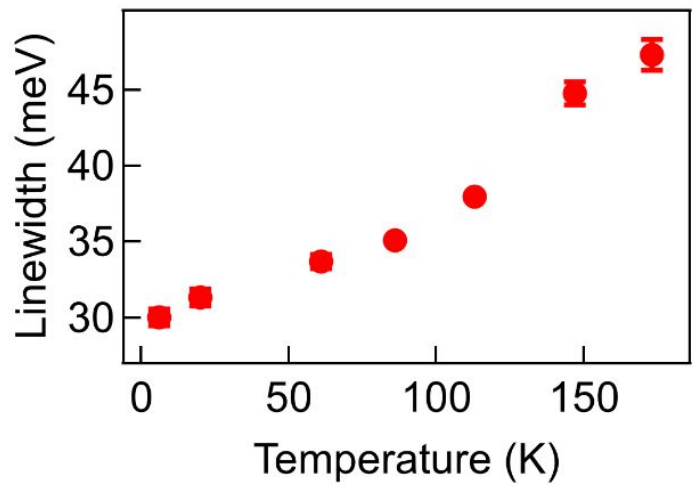

Figure S9. Temperature dependence of trion linewidth. The linewidths of the trions are extracted from the PL spectra in the main text.

\section{Supplementary References}

1. Cheng, G. H.; Li, B. K.; Zhao, C. Y.; Yan, X.; Wang, H.; Lau, K. M.; Wang, J. N. Interfacially Bound Exciton State in a Hybrid Structure of Monolayer $\mathrm{WS}_{2}$ and InGaN Quantum Dots. Nano Lett. 2018, 18, (9), 5640-5645.

2. $\quad$ Kulig, M.; Zipfel, J.; Nagler, P.; Blanter, S.; Schüller, C.; Korn, T.; Paradiso, N.; Glazov, M. M.; Chernikov, A. Exciton Diffusion and Halo Effects in Monolayer Semiconductors. Phys. Rev. Lett. 2018, 120, (20), 207401.

3. Butov, L. V.; Ivanov, A. L.; Imamoglu, A.; Littlewood, P. B.; Shashkin, A. A.; Dolgopolov, V. T.; Campman, K. L.; Gossard, A. C. Stimulated scattering of indirect excitons in coupled quantum wells: Signature of a degenerate Bose-gas of excitons. Phys. Rev. Lett. 2001, 86, (24), 5608-5611.

4. Sze, S. M.; Ng, K. K., Physics of semiconductor devices. 3rd ed.; Wiley-Interscience: Hoboken, NJ, 2007.

5. $\quad$ Moody, G.; Dass, C. K.; Hao, K.; Chen, C. H.; Li, L. J.; Singh, A.; Tran, K.; Clark, G.; Xu, X. D.; Berghäuser, G.; Malic, E.; Knorr, A.; Li, X. Q. Intrinsic homogeneous linewidth and broadening mechanisms of excitons in monolayer transition metal dichalcogenides. Nat. Commun. 2015, 6, 8315.

6. Cadiz, F.; Courtade, E.; Robert, C.; Wang, G.; Shen, Y.; Cai, H.; Taniguchi, T.; Watanabe, K.; Carrere, H.; Lagarde, D.; Manca, M.; Amand, T.; Renucci, P.; Tongay, S.; Marie, X.; Urbaszek, B. Excitonic Linewidth Approaching the Homogeneous Limit in $\mathrm{MoS}_{2}$-Based van der Waals Heterostructures. Phys. Rev. X 2017, 7, (2), 021026. 
7. Selig, M.; Berghäuser, G.; Raja, A.; Nagler, P.; Schüller, C.; Heinz, T. F.; Korn, T.; Chernikov, A.; Malic, E.; Knorr, A. Excitonic linewidth and coherence lifetime in monolayer transition metal dichalcogenides. Nat. Commun. 2016, 7, 13279. 